\title{
La odontología forense en las Fuerzas Armadas: Una asignatura pendiente
}

\author{
Bel Blesa A. ${ }^{1}$
}

Sanid. mil. 2011; 67 (4): 375-380; ISSN: 1887-8571

\begin{abstract}
RESUMEN:
La odontología forense es una disciplina que está abriéndose paso en nuestras Fuerzas Armadas en respuesta a necesidades derivadas de la participación de España en misiones internacionales y al cumplimiento de lo acordado en el marco de la OTAN. Aún queda mucho camino que recorrer en lo referente a la identificación humana. Se analiza la situación actual referente a la Ficha de Identificación Odontológica en vigor y propone un nuevo modelo más acorde a los estándares internacionales.
\end{abstract}

PALABRAS CLAVE: Odontología forense; Ficha Dental; Odontograma; Identificación Humana.

\section{Forensic dentistry in the Armed Forces: an unresolved matter}

SUMMARY:

Forensic dentistry is an emerging discipline in our Armed Forces that answers the needs derived from the Spanish participation in overseas deployments and NATO agreements. There is still much to be done as far as human identification is concerned. The current Dental Identification Card is evaluated and a new model more in accordance with international standards is proposed.

KEY WORDS: Forensic dentistry, Dental records, Dental chart, Human Identification.

\section{INTRODUCCIÓN}

Las Fuerzas Armadas españolas han sido objeto de un cambio cualitativo y cuantitativo en los últimos 20 años. La participación activa en misiones de ayuda humanitaria y de mantenimiento de la paz formando parte de contingentes de las Naciones Unidas, OTAN y UE ha sido el catalizador de este, por otro lado, necesario cambio.

Se ha producido un proceso de profesionalización de las fuerzas armadas de manera que el multitudinario ejército de reemplazo ha sido sustituido por un ejército muy inferior en número pero muy superior en formación, profesionalidad y medios técnicos.

La racionalización de las fuerzas armadas no afecta sólo a la Fuerza, sino también a las estructuras satélites que permiten la operatividad de la misma: el Apoyo a la Fuerza.

Como no podía ser de otro modo, la Sanidad Militar se ve afectada directamente por estos cambios que, en primer lugar, se manifiestan con la reorganización de la Red Hospitalaria de la Defensa. Se reestructuran los centros sanitarios, se transforman o simplemente desaparecen, obedeciendo a la racionalización de la Red Sanitaria Militar.

Por otro lado se produce un enfoque diferente de la Sanidad Militar en relación a tiempos pretéritos. Las funciones principales y el sentido de la Sanidad Militar actual son las funciones LogísticoOperativa y Pericial, quedando en un segundo plano la función asistencial, que hasta ahora había predominado.

Cte. Odontólogo. Hospital Militar O’Donnell. Servicio de Odontología. Ceuta. España.

Dirección para correspondencia: abelble@oc.mde.es

Recibido: 4 de enero de 2011

Aceptado: 7 de marzo de 2011
Como parte relevante de la función pericial emerge lo referente a la identificación humana.

Se entiende por identificación humana al proceso por el cual se determinan las características individualizadoras de un sujeto de manera que éste sea único y diferente dentro del conjunto de la población. No hay dos personas idénticas. Incluso entre gemelos univitelinos se expresan rasgos que los diferencian entre sí.

La identificación humana plantea una serie de problemas de diversa índole. Tiene repercusiones legales en asuntos de herencias, de tutoría de menores, de seguros de vida; tiene repercusiones psicológicas para familiares y allegados de desaparecidos (se prolonga la agonía de la espera y la búsqueda infructuosa); tiene repercusiones sociales y políticas (un claro ejemplo serían las fosas comunes durante la Guerra Civil española, o el caso del accidente en Turquía del Yakolev-42), etc.

Debido a la participación de nuestras Fuerzas Armadas en conflictos bélicos y desastres naturales, la problemática de la identificación humana ha irrumpido en el seno del ejército. Es inevitable que sucedan accidentes, atentados y acciones hostiles, que en un momento dado requieran la activación de los mecanismos forenses encaminados a la identificación.

En el recuerdo de todos los españoles están los desafortunados sucesos acontecidos a compañeros militares y civiles en el cumplimiento de su deber fuera de nuestras fronteras. Algunos de estos hechos evidenciaron la carencia de unos medios necesarios para afrontar con éxito las tareas de identificación. Éste es el punto de inflexión a partir del cual se comienza a legislar y se establece el protocolo de actuación y colaboración en lo referente a catástrofes con víctimas múltiples (R.D. 32/2009) ${ }^{1}$. En este Real Decreto la fase de identificación adquiere relevancia.

A nivel de las Fuerzas Armadas se crea la Unidad de Identificación, que centraliza la totalidad de las Fichas de Identificación 
Sanitarias (FISAN), reguladas mediante la Instrucción Técnica de la Inspección General de Sanidad de 7 de febrero de $2006^{2}$.

Un componente fundamental de la FISAN es la Ficha Dental, que tiene la finalidad de reflejar los datos de carácter odontológico con valor forense relacionados con la identificación. De esta manera y respondiendo a una necesidad imperiosa, se comienza a vislumbrar la utilidad de una especialidad de la odontología que hasta ahora se había mantenido en un segundo plano; la odontología forense.

\section{Odontología forense y odontología pericial}

Los términos odontología forense y odontología pericial se emplean a menudo indistintamente, sin embargo, hay matices que los diferencian en su esencia.

En el entorno de nuestras Fuerzas Armadas, la función pericial de la odontología se ha desarrollado fundamentalmente en la determinación de APTITUDES y en la valoración de lesiones y daños en el área bucodental. Ambas tareas consisten en la aplicación de unos baremos establecidos de forma arbitraria concebidos para clasificar los grados de aptitud para una actividad o tarea determinadas. (R.D. 944/2001; O.M. 282/82; O.M. 74/1992; Resolución 452/38009/2002-BOD no 42) ${ }^{3-6}$.

Esta función pericial puede desarrollarla cualquier odontólogo generalista (o médico estomatólogo) sin más formación que la propia que le confiere su titulación y el conocimiento del baremo que debe aplicar en cada caso ${ }^{7}$.

En nuestro ejército, la odontología forense tiene un significado diferente al pericial en la medida en que se pretende hacer mención únicamente a la parte de esta disciplina que trata el tema de la identificación humana.

Si bien la odontología pericial no requería de ningún conocimiento más allá de los propios de la licenciatura (o especialidadestomatología-), con la odontología forense no ocurre así. Se habla ya de una «especialidad» de la odontología (entrecomillo la palabra porque a día de hoy en España no se contemplan especialidades en esta licenciatura, al contrario que en otros países europeos o americanos), que como tal, ha desarrollado una serie de conocimientos y disciplinas que se escapan del contenido propio de la licenciatura, de manera que la odontología forense debe ser realizada por licenciados en odontología (o médicos especialistas en estomatología) con conocimientos especializados en la materia ${ }^{8}$. En la actualidad la universidad española cuenta con cursos de formación (Cursos de Experto en Odontología Forense, Master en Odontología Legal y Forense) y con cursos de información (Cursos online, cursos de formación continuada, etc.).

Nuestra Sanidad Militar cuenta en la actualidad con un número muy limitado de odontólogos con formación reglada en odontología forense obtenida por medios propios. Por otro lado, hay algunos odontólogos y médicos estomatólogos con cursos de información en odontología forense y cursos de información en odontología legal (más enfocados al área de lo pericial).

Debido a la trascendencia legal, psicológica, política y social que entrañan los problemas de identificación humana, la odontología forense debe ser realizada por odontólogos (o médicos estomatólogos) con formación especializada en esta disciplina (serían los «odontólogos forenses», figura aún inexistente en España, pero muy

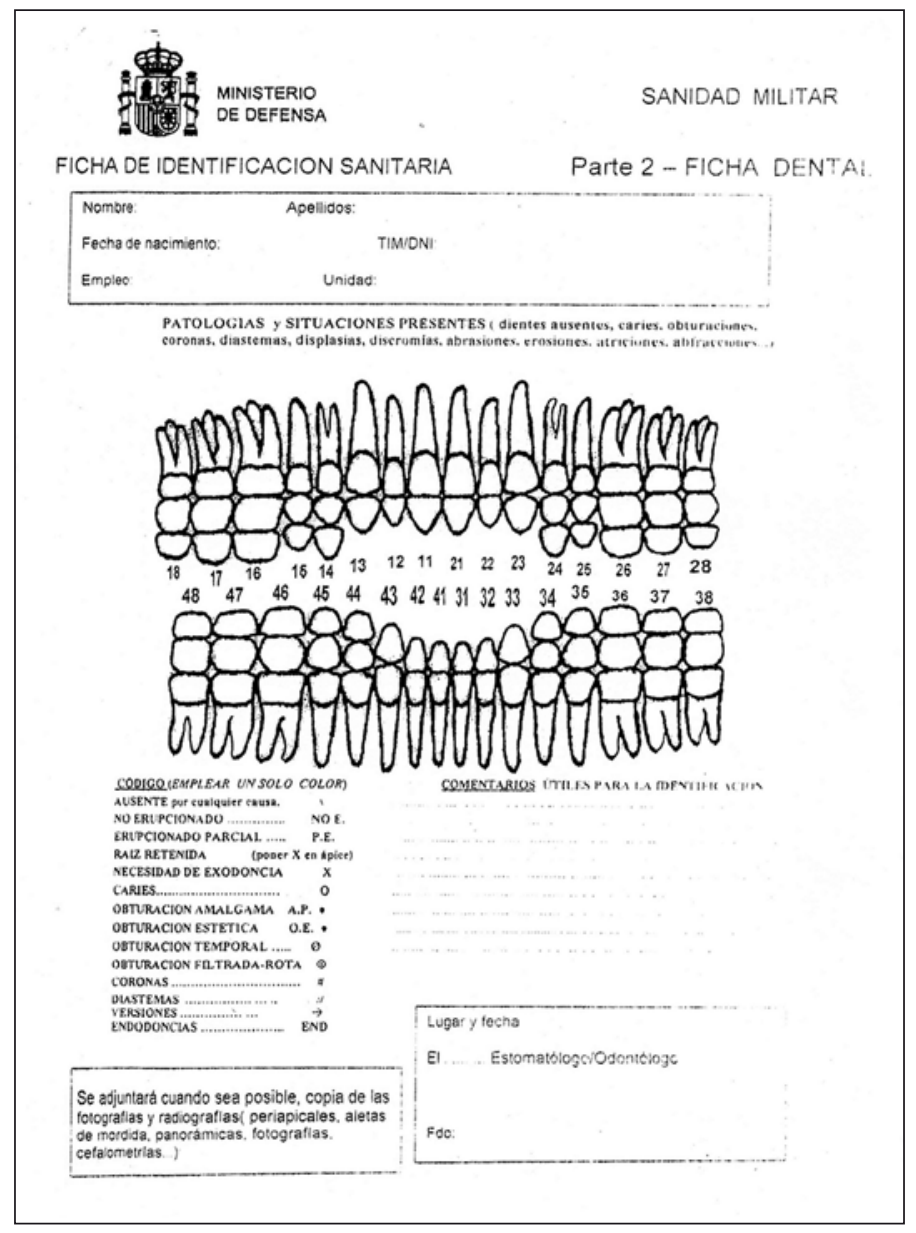

Figura 1. Ficha Dental vigente.

extendida ya en países como Francia, Alemania, Suecia, Estados Unidos, Reino Unido, Argentina, México, Colombia, Chile, etc.)

Se debe potenciar la formación de los odontólogos y estomatólogos militares, como mínimo, ofreciéndoles cursos de información en odontología forense, para que se pueda realizar con mayor eficacia una de las labores encomendadas a este colectivo de la Sanidad Militar que es la confección de la Ficha Dental con fines de identificación.

\section{Ficha Dental con fines de identificación: necesidad imperiosa de cambio}

Actualmente contamos con un modelo de Ficha Dental de tipo anatómico en el que se anotan una serie de códigos que hacen referencia a algunos tratamientos y patologías. (Figura 1). Ese modelo resulta obsoleto y poco eficaz para el objetivo pretendido.

Existen otros diseños más adecuados de reconocida utilidad a nivel internacional. Tal es el modelo adoptado por la INTERPOL, que sustituye la representación anatómica de los dientes por una interpretación geométrica, en beneficio de la objetividad y de la posibilidad de informatización ${ }^{9,10}$.

Dada su probada eficacia, se recomienda un odontograma del tipo de la INTERPOL y a ser posible, con la modificación incorporada por la AKFOS (Academia Alemana de Odonto-Estomatología 
Forense) consistente en la inclusión en el esquema de las zonas radiculares. (Figura 2).

Debemos simplificar en la medida de lo posible el diseño del odontograma sin perder el objetivo que se pretende, que es lograr una identificación con los datos registrados.

Cuando se recogen datos en un odontograma, si se hace bajo el punto de vista del odontólogo general o estomatólogo, normalmente se señalan las patologías que hay presentes y los tratamientos que le vamos realizando. En raras ocasiones se ven odontogramas donde se apunte la totalidad de tratamientos que traiga realizados el individuo en otros centros odontológicos.

Cuando un odontólogo forense registra los datos en un odontograma presta atención al detalle. Toma nota de todo aquello que pueda servir como rasgo individualizador de esa persona. Se considera todo lo normal y todo lo extraordinario o anómalo. A mayor grado de anormalidad en el rasgo registrado, mayor capacidad individualizadora ${ }^{11,12}$

Un odontograma concebido para la identificación humana debe contar con elementos que permitan su clasificación para facilitar la búsqueda de posibles identidades. Este importante paso en los procedimientos de identificación humana en casos múltiples se facilita en gran medida con la incorporación de las aplicaciones informáticas diseñadas al efecto. $\left(\mathrm{WIN}-\mathrm{ID}^{\circledR}{ }^{\circledR}, \mathrm{PLASS} \mathrm{DATA}{ }^{\circledR}, \mathrm{CAPMI}^{\circledR}, \ldots\right)^{13}$.

La información contenida en el odontograma debe ser clara e inequívoca, por ello es aconsejable emplear códigos normalizados. Existen muchos sistemas de códigos. Todos son válidos; sin embargo se prefieren aquellos más intuitivos porque simplifican la labor de registro de datos. En cualquier caso, siempre debe incluirse en la Ficha Dental una leyenda con el significado de los códigos utilizados.

Debido al gran número de tratamientos, patologías y anomalías que pueden presentarse en la boca, es materialmente imposible desarrollar una codificación que abarque el conjunto de aspectos que pueden darse y que además cumpla con el requisito de la sencillez y la claridad. Es por esto por lo que la mayoría de las codificaciones incluyen sólo los rasgos más comunes y las Fichas Dentales cuentan con unas zonas de libre escritura donde reflejar las aclaraciones oportunas o anotar los rasgos que no tienen representación en el código empleado.

Otro aspecto a considerar respecto al formato de Ficha Dental que se emplea actualmente en las Fuerzas Armadas es su enfoque dual de manera que se utiliza para recoger datos odontológicos con fines de identificación y además se pretende también que sirva como documento que dictamine la APTITUD del personal militar con ocasión de su despliegue fuera del Territorio Nacional.

Ya se han comentado las limitaciones de esta ficha para el objetivo forense y en el apartado correspondiente se propone un nuevo modelo de Ficha Dental, por supuesto también abierto a debate constructivo, pero más acorde con los estándares forenses aceptados por la comunidad científica internacional.

Es imperativo desglosar esa dualidad actual. Una cosa es determinar la APTITUD dental basándose en unos baremos y criterios del todo arbitrarios (sería otro tema amplio para debatir) y otra cosa es recopilar datos odontológicos con fines forenses. No deben mezclarse los conceptos porque va en detrimento de una correcta cumplimentación de la Ficha Dental, prestándose mucha más atención en ocasiones a dictaminar la APTITUD que a tener como objetivo la identificación del individuo, resultando finalmente una Ficha Dental inútil para los propósitos para los que está concebida en esencia ${ }^{14}$.

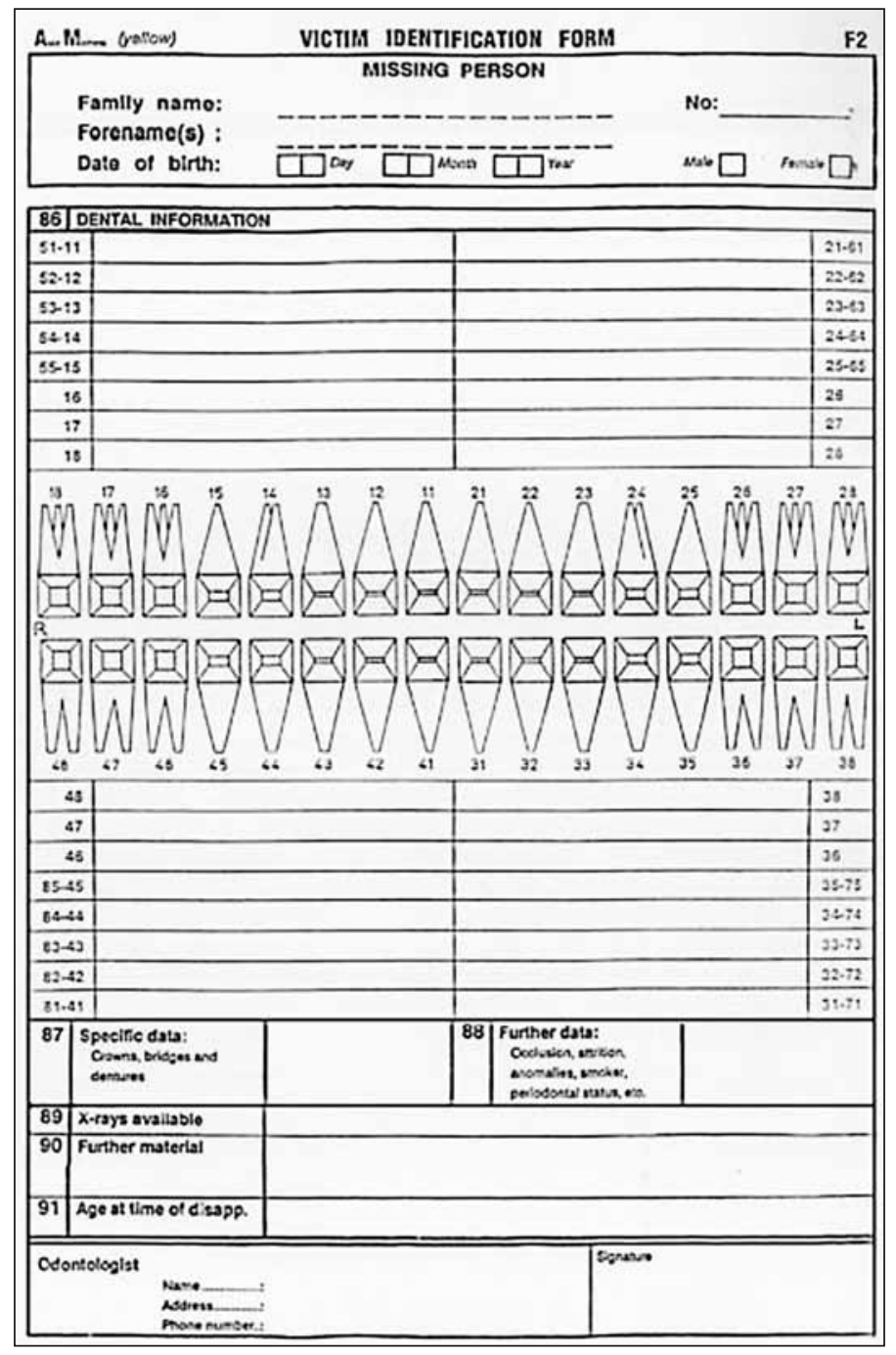

Figura 2. Odontograma de la AKFOS.

\section{Nuevas Tecnologías en las Fuerzas Armadas: Ficha Dental Forense informatizada}

La implantación de las nuevas tecnologías en las Fuerzas Armadas está suponiendo un avance importante hacia el modelo de ejército del siglo XXI.

En la Sanidad Militar hay proyectos en marcha para informatizar los datos sanitarios del personal militar, entre otros objetivos (Proyecto Balmis).

La odontología forense militar tiene la gran oportunidad de formar parte de este proyecto con la informatización de la Ficha Dental y demás registros odontológicos (fotografías, radiografías, etc.). De esta manera se posibilitaría la centralización digital de los datos que, con los debidos filtros de seguridad, podrían ser consultados y actualizados en tiempo real desde puestos periféricos conectados en red. Con esto se agilizaría el proceso, evitando la duplicidad de los datos.

Los datos deben ir codificados de una manera que permita su almacenamiento sin ocupar un espacio tal que ralentice la aplicación o genere problemas de capacidad. Actualmente existen artículos publicados que forman parte de un trabajo de investigación aún sin concluir que trata este asunto de la codificación y que podría ser interesante para nuestra Sanidad Militar su consideración. Se trata 

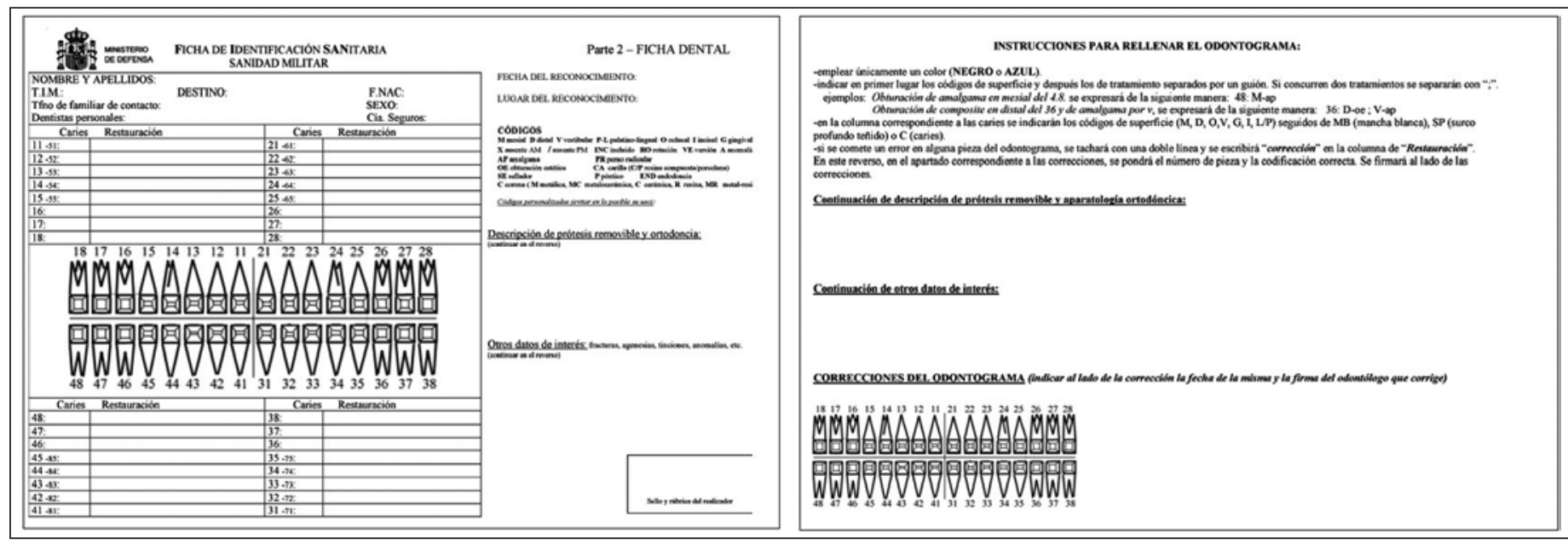

Figura 3. Ficha Dental propuesta. Anverso y reverso

de un trabajo muy elaborado y con conocimientos profundos de la odontología forense. (Martínez J, Luna JD, Valenzuela A: La variabilidad de los tratamientos dentales en una población militar española y su importancia para la estimación de la probabilidad de identificación dental. Cuad Med Forense 2008; 14 (53-54): 223-233 $3^{15,16}$.

El panorama es prometedor, sin embargo, siendo realistas, aún queda tiempo para ver materializada la Ficha dental informatizada. A día de hoy es preceptivo continuar con el protocolo actual en so- porte papel, que espero se consiga mejorar dándole un enfoque predominantemente forense y no clínico como ocurre en la actualidad.

\section{Propuesta de Ficha Dental para las Fuerzas Armadas}

Una vez analizadas las limitaciones y carencias encontradas en la Ficha Dental vigente en nuestro Ejército desde el punto de vista

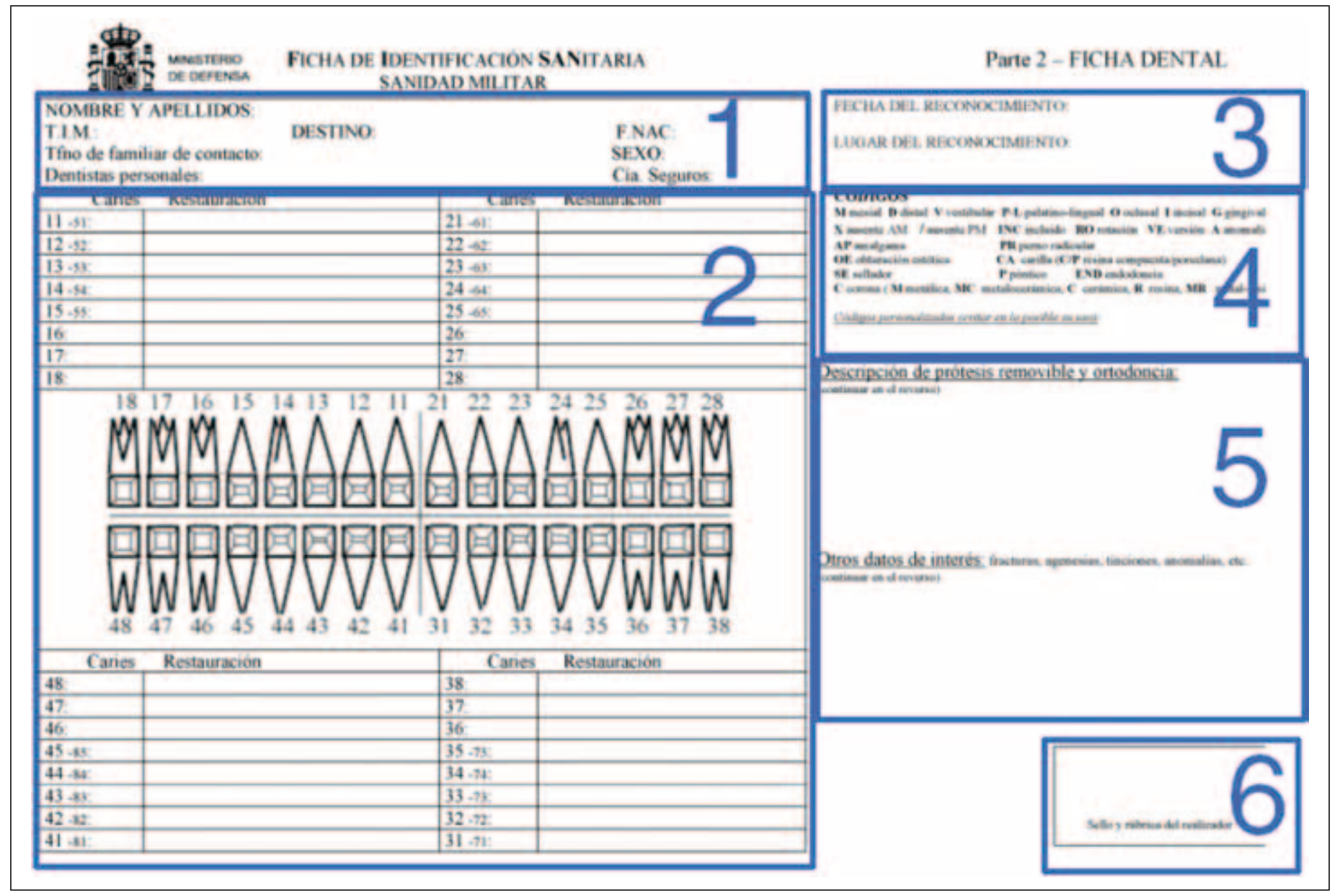

Figura 4. Anverso de la Ficha Dental propuesta. 
de la odontología forense, se propone un nuevo formato que está concebido desde esta perspectiva. (Figura 3).

La Ficha Dental propuesta se estructura en varias áreas:

\section{ANVERSO (Figura 4).}

1. Espacio para los datos de filiación y de interés forense para facilitar la clasificación: fecha de nacimiento (edad) y sexo. También se incorpora un campo para indicar los dentistas que le han tratado. De esta manera, se tiene una fuente adicional de datos donde conseguir registros del individuo llegado el caso. Registrar la compañía de seguros del sujeto también puede proporcionar un camino para obtener datos antemortem.

2. Espacio del odontograma: éste es de tipo esquemático, con espacio reservado a cada diente para anotar los códigos oportunos.

3. Fecha y lugar del reconocimiento: nos indicará la «antigüedad» de los datos registrados y la posibilidad de que éstos hayan podido variar en función del tiempo transcurrido.

4. Espacio reservado a la leyenda de los códigos empleados.

5. Espacio de libre escritura: se anotarán los hallazgos que no tengan representación en los códigos, se describirán las prótesis o aparatos de ortodoncia, etc.

6. Firma del odontólogo o estomatólogo que toma los datos: no debemos olvidar que estamos ante un documento con vali- dez legal que contiene datos personales sujetos a la vigente LOPD (Ley Orgánica de Protección de Datos de Carácter Personal 15/1999).

\section{REVERSO (Figura 5)}

1. Espacio donde se exponen las instrucciones para rellenar el odontograma.

2. Espacio reservado para continuar con lo establecido en el espacio $n^{\circ} 5$ del anverso.

3. Espacio reservado a anotar las correcciones que se efectúen.

\section{CONCLUSIONES}

La implantación de la odontología forense en nuestras Fuerzas Armadas exige la adopción de un nuevo modelo de Ficha Dental acorde con los estándares científicos internacionales. La Ficha Dental dual no resulta operativa para los objetivos forenses.

Es necesario proporcionar a los odontólogos militares cursos de información en odontología forense orientados a la identificación humana; sin embargo, las labores especializadas de odontología forense deben ser realizadas por odontólogos con formación específica en dicha materia.

La odontología forense es una labor de equipo; aunando esfuerzos y trabajando conjuntamente se pueden alcanzar los objetivos

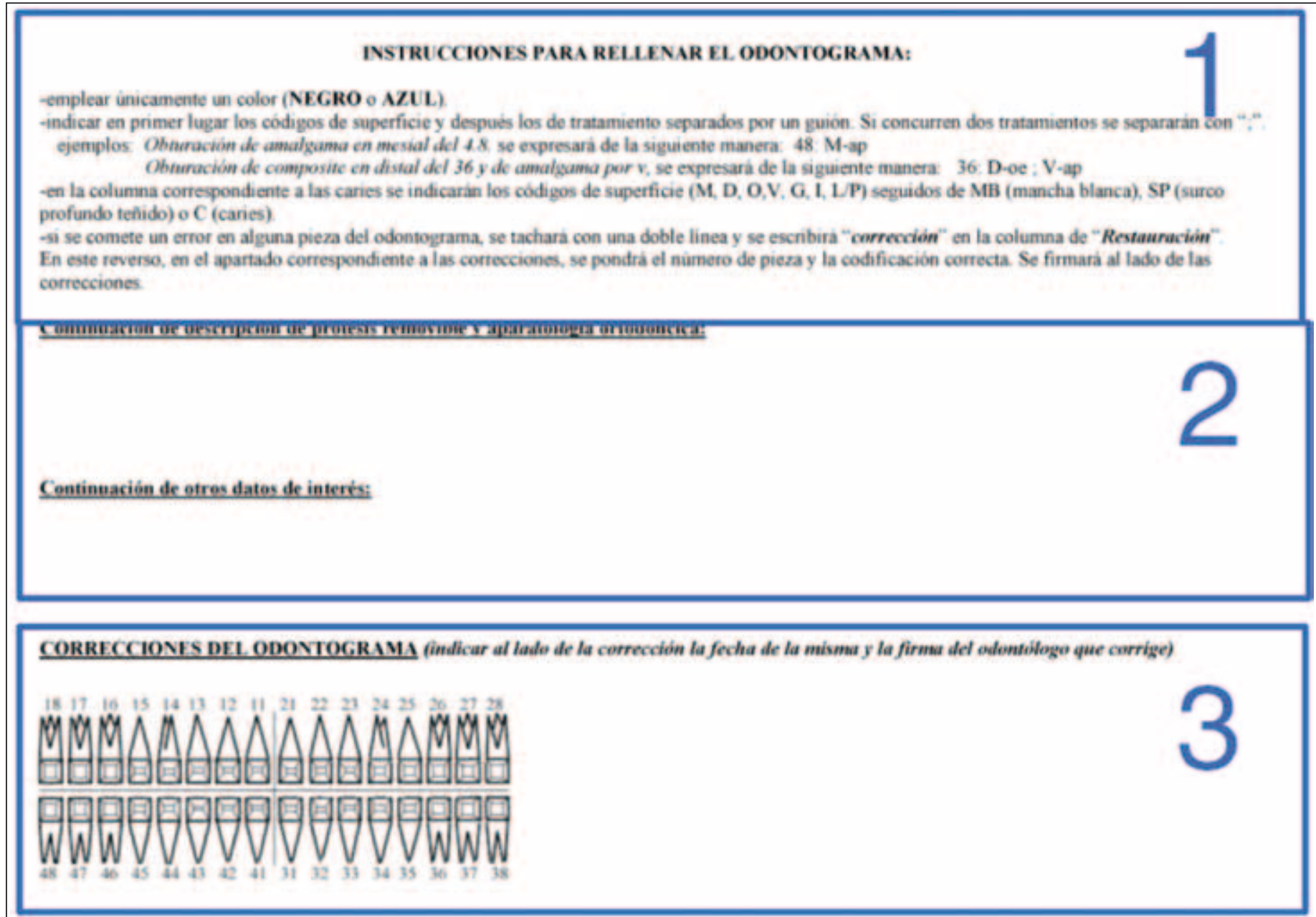

Figura 5. Reverso de la Ficha Dental propuesta. 


\section{A. Bel Blesa}

pretendidos con la Ficha Dental y los compromisos adquiridos con la ratificación del STANAG 2464 (equipos de identificación de víctimas, equipamiento básico, colaboración entre países, etc.)

\section{BIBLIOGRAFÍA}

1. Real Decreto 32/2009: Protocolo de actuación Médico Forense y de policía Científica en sucesos con víctimas múltiples.

2. Instrucción Técnica de 7 de febrero de 2006 de la IGESAN sobre la Ficha de Identificación Sanitaria y Huella Genética.

3. Real Decreto 944/2001 de 3 de agosto por el que se aprueba el Reglamento para la determinación de la aptitud psicofísica del personal de las Fuerzas Armadas.

4. Orden Ministerial 282/82 de 20 de octubre (Diario oficial de marina $\mathrm{n}^{\circ} 251$ ): para la determinación de la aptitud para el personal de buceo.

5. Orden Ministerial 74/1992 de 14 de octubre, por la que se aprueban las normas para la evaluación psicofísica del personal de las Fuerzas Armadas con responsabilidad de vuelo.

6. Resolución 452/38009/2002 de 20 de febrero de la Subsecretaría, por la que se convoca el proceso selectivo para cubrir las plazas para acceso a Militar Profesio- nal de Tropa y Marinería (BOD 42 de 28 de febrero de 2002).

7. Protocolo de 26 de julio de 2006 de la IGESAN sobre reconocimiento odontológico básico y formalización de la Ficha Dental.

8. STANAG 2464 MED: THE MILITARY DENTAL FIELD IDENTIFICATION SERVICES.

9. Disaster Victim Identification (DVI) Guide (www.interpol.int)

10. Ante-Mortem (Yellow) VI Form (www.interpol.int/Public/DisasterVictim/Forms/ Default.asp)

11. ABFO Guidelines for the Use of Dental Information in Missing Person and Unidentified Body Cases (www.abfo.org/id mark guidelines.htm)

12. ABFO Body Identification Guidelines (www.abfo.org/id_mark_guidelines.htm)

13. Edward E. Herschof et al. Manual of Forensic odontology $4^{\text {th }}$. Ed. ASFO.

14. Bel A: Proposal of an odontological identification file for the armed forces. Revue Internationale des Services de Santé des Forces Armeés (RISSFA) 2009; 82, $\mathrm{n}^{\circ}$ $1(53-54): 3-11$

15. Martínez J, Luna JD, Valenzuela A: La variabilidad de los tratamientos dentales en una población militar española y su importancia para la estimación de la probabilidad de identificación dental. Cuad Med Forense 2008; 14 (53-54): 223-233.

16. Hernández MA, Martínez J: La asistencia pericial odontológica en la identificación de cadáveres en grandes catástrofes. San Mil 2007;63(4):287-290. 